\title{
POLARIMETRIC DECOMPOSITION FOR FOREST BIOMASS RETRIEVAL
}

\author{
Stefan Sauer, Florian Kugler, Seung-Kuk Lee, Kostas Papathanassiou \\ German Aerospace Center (DLR), Microwaves and Radar Institute (HR), Germany
}

\begin{abstract}
In this paper, the decomposition of fully polarimetric SAR data into three canonical scattering mechanisms is investigated with respect to forest biomass retrieval. It is demonstrated that the dominant backscattering contribution depends strongly on the frequency, incidence angle, and stand condition. The relation between in-situ biomass measurements and the individual decomposition components is examined.
\end{abstract}

Index Terms - Forest SAR remote sensing, biomass retrieval, polarimetric decomposition.

\section{INTRODUCTION}

In the past, it has been proposed to estimate forest biomass by measuring Synthetic Aperture Radar (SAR) backscattering at low frequencies and relating the intensity of different polarization channels to biomass [1, 2]. Primarily, HV- and/or HHpolarized backscatter depend on the biomass level. P-band backscatter has larger dynamic range and higher saturation level than L-band backscatter [1,2].

In this paper, a decomposition of polarimetric SAR data $[4,3]$ into three canonical scattering mechanisms (volume, dihedral, and direct surface reflection) is investigated in order to enhance the biomass estimation. First, for measurements over a tropical peat swamp forest (Mawas test site), the decomposition results obtained from P-band and L-band data are compared and the influence of the incidence angle is investigated. Subsequently, the backscattering power of the three components are related to in-situ biomass measurements for a boreal forest test site (Krycklan) at L-band and P-band.

\section{POLARIMETRIC DECOMPOSITION}

The polarimetric decomposition is based on the model introduced in [4] that includes three scattering mechanisms, namely canopy (or volume), double-bounce, and surface scattering. In particular, the generalized Freeman-Durden decomposition - a hybrid Freeman/eigenvalue technique is used for avoiding negative powers in all contributions by keeping only the non-negative eigenvalue spectrum [3].
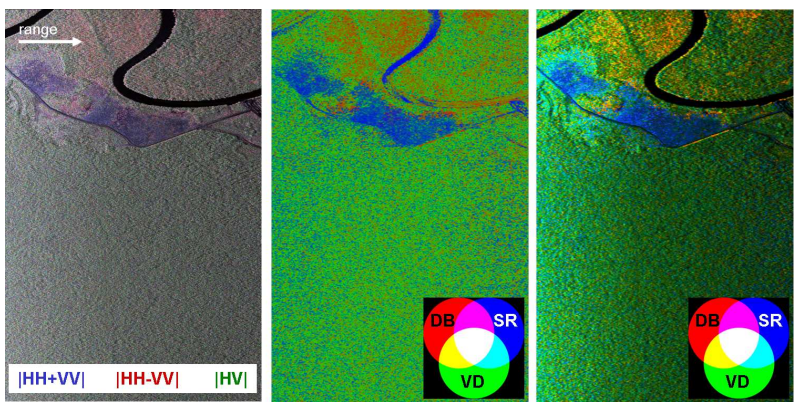

Fig. 1. Mawas test site at L-band. Left: POLSAR image, middle: Normalized polarimetric decomposition, right: Decomposition including power.

\section{EXPERIMENTAL RESULTS}

\subsection{Polarimetric Decomposition Over Tropical Forest}

First, the polarimetric decomposition is applied to POLSAR images of a tropical forest (Mawas test site) over flat ground. Figures 1 and 2 show the Pauli color-coded images, the normalized decompositions and the decompositions including the power at L- and P-band, respectively. As anticipated, over tropical forest the volume diffusion is dominant at L-band and the dihedral and surface backscattering contributions are rather weak. At P-band, direct surface reflection prevails in near range and diminishes with range, whereas doublebounce increases at far range. This analysis is enhanced by figure 3 where the percentage of the scattering components averaged in the azimuth direction vs. the incident angle is depicted. Both at L- and P-band, the volume diffusion is nearly constant over range, for L-band at around 60 percent and for P-band at approximately 35 percent. In near range, the surface reflection component is dominant with more than 50 percent at $\mathrm{P}$-band, and decreases with range to around 30 percent in far range. The behavior of the dihedral contribution is opposite, i.e., it starts at 10 percent in near range and increases to more than 30 percent in far range. Similar, but less pronounced effects can be appreciated for L-band. This implies that direct surface reflection is dominant at P-band for look angles between 25 and $30 \mathrm{deg}$ that shall be employed in the BIOMASS mission. 

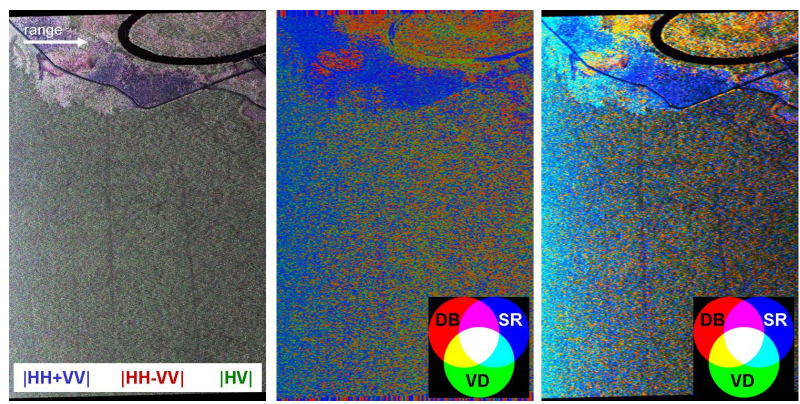

Fig. 2. Mawas test site at P-band. Left: POLSAR image, middle: Normalized polarimetric decomposition, right: Decomposition including power.

\subsection{Biomass Retrieval Over Boreal Forest}

Figure 4 illustrates the Pauli image and the polarimetric decomposition at P-band of the test site of Krycklan, a boreal forest over terrain with large topography variations. It can be noticed that volume diffusion is very weak and surface and double-bounce scattering is prevalent. The biomass measured in-situ for 31 stands ranges from 23 to 183 tons per ha (see figure 5). Inside most of the stands having constant biomass values, several polarimetric contributions are present.

Figure 6 shows the correlation between biomass and $\gamma_{0}$ of the polarimetric channel HV and of the dihedral and volume components. It can be observed that the dynamic range in the dihedral contribution is very large compared to the polarimetric channels and the surface and volume components. Furthermore, the double-bounce correlation does not seem to saturate for rising biomass levels. The pattern between the surface component and biomass seems to follow a random distribution. It is clear that the mapping for the correlation between the dihedral contribution and biomass and the mapping of the correlation between the volume diffusion and biomass are quite diverse.

The same investigations have been performed for a dataset over Krycklan forest acquired from the opposite viewing angle. The plots of $\gamma_{0}$ of the HV channel and of $\gamma_{0}$ of the polarimetric descriptors vs. biomass (figure 8) confirm largely the results obtained previously from the opposite direction.

At L-band (see figure 7) volume reflection is significantly stronger than at P-band. Again stands with constant biomass (figure 9) are characterized by heterogeneous backscattering phenomena.

Figure 10 shows the correlation between biomass and $\gamma_{0}$ of the polarimetric channel HV and of the dihedral and volume component in L-band. It is evident that the dynamic range is wider for the double-bounce contribution than for the polarization channel and the volume component. It seems that saturation occurs at around 120 tons/ha.

In principle, these observations are corroborated by the analysis performed on the dataset acquired from the opposite
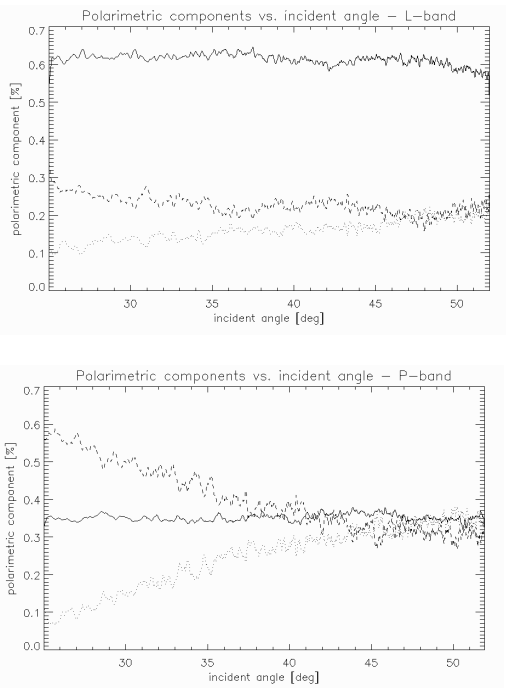

Fig. 3. Mawas test site. Polarimetric components in percentage vs. incident angle for L-band (top) and P-band (bottom): Volume (solid line), surface (dashed line), dihedral (dotted line).
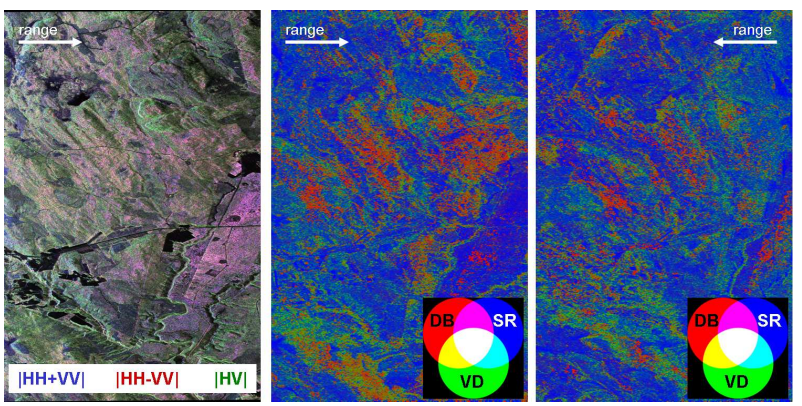

Fig. 4. Krycklan test site at P-band. Left: POLSAR image, middle: Normalized polarimetric decomposition, right: Decomposition from opposite viewing angle.
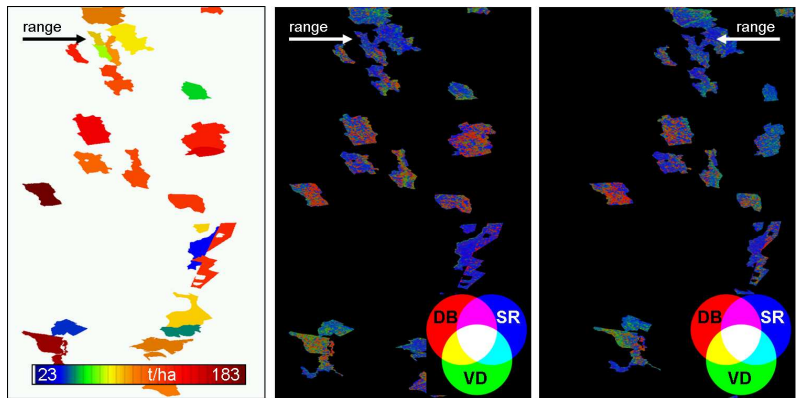

Fig. 5. Krycklan test site at P-band. Left: Biomass stands, middle: Normalized polarimetric decomposition for biomass stands, right: Decomposition from opposite viewing angle. 

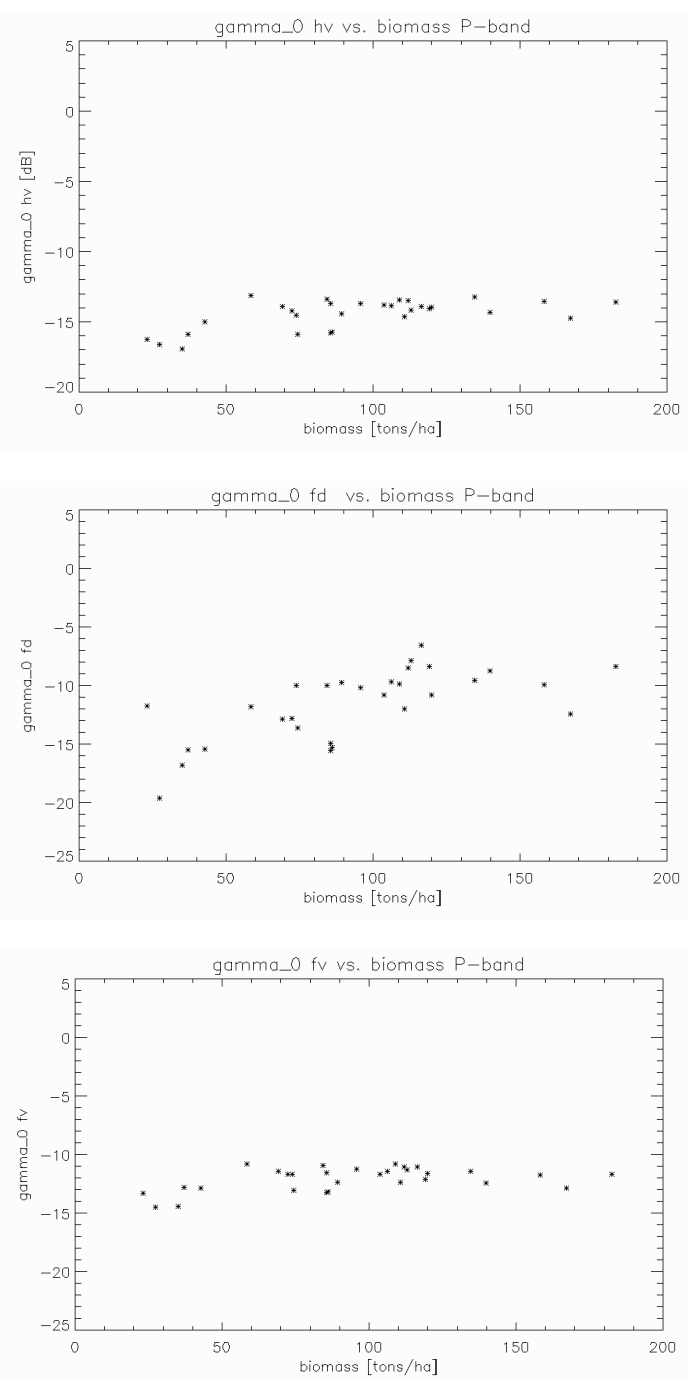

Fig. 6. Krycklan test site at P-band - $\gamma_{0}$ vs.biomass. Top: HV channel, middle: Dihedral, bottom: Volume diffusion.
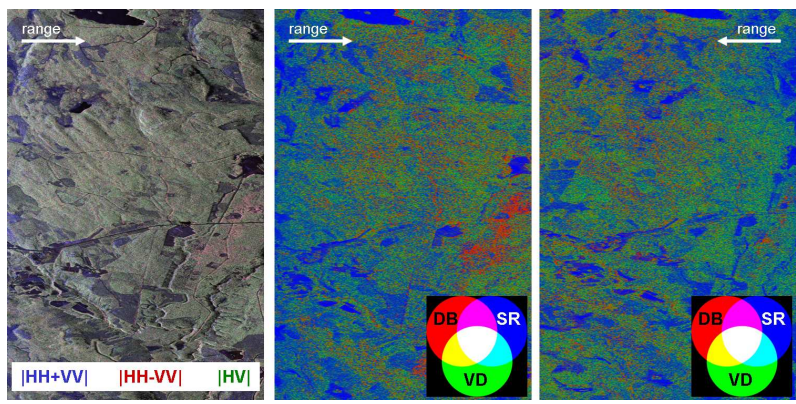

Fig. 7. Krycklan test site at L-band. Left: POLSAR image, middle: Normalized polarimetric decomposition, right: Decomposition from opposite viewing angle.
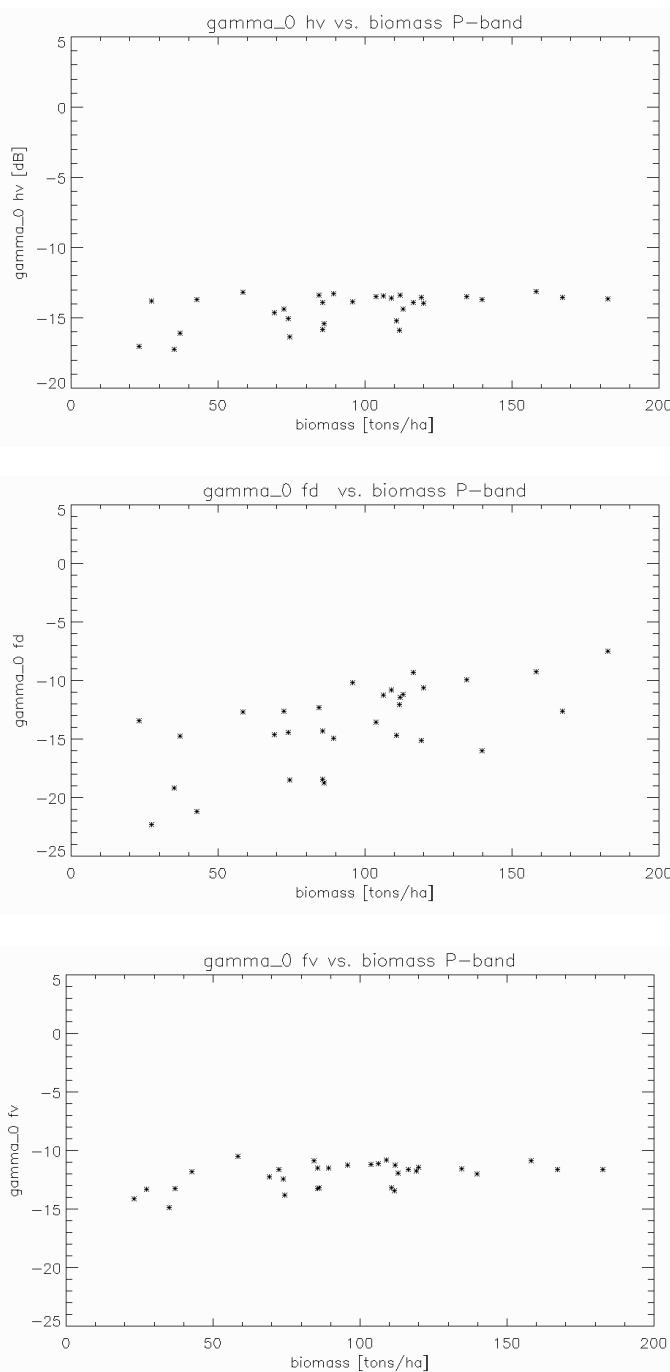

Fig. 8. Krycklan test site at P-band acquired from opposite viewing angle - $\gamma_{0}$ vs. biomass. Top: HV channel, middle: Dihedral, bottom: Volume diffusion.
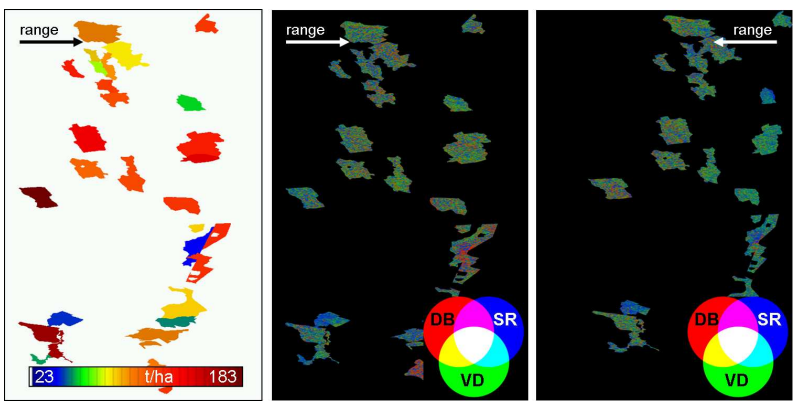

Fig. 9. Krycklan test site at L-band. Left: Biomass stands, middle: Normalized polarimetric decomposition for biomass stands, right: Decomposition from opposite viewing angle. 

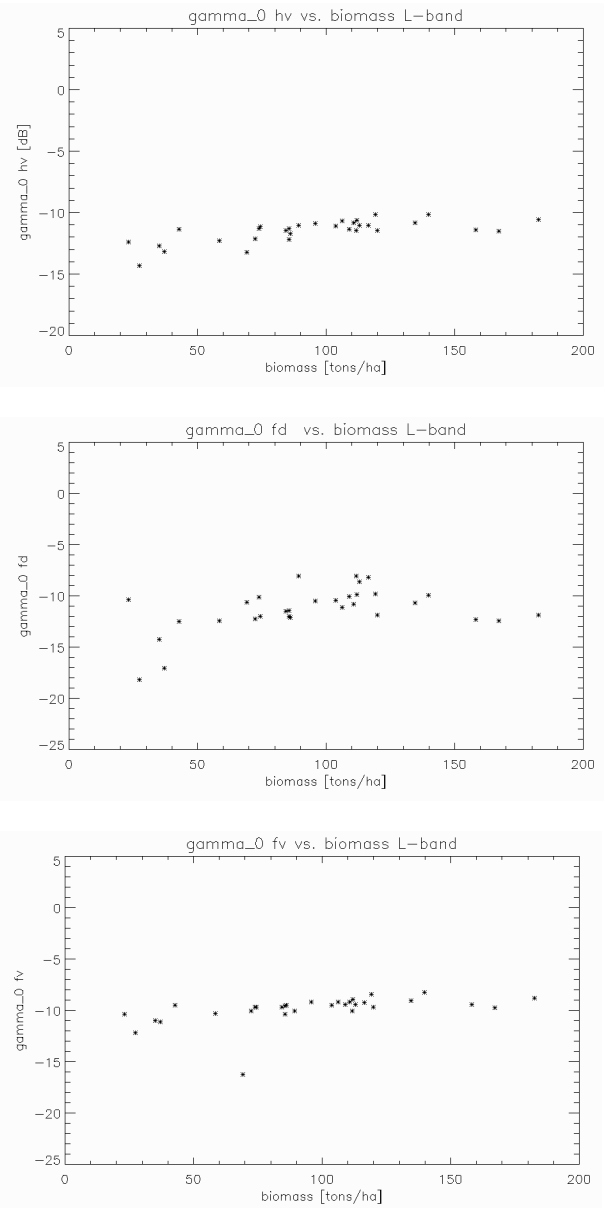

Fig. 10. Krycklan test site at L-band - $\gamma_{0}$ vs.biomass. Top: HV channel, middle: Dihedral, bottom: Volume diffusion.

viewing angle (figure 11).

\section{CONCLUSIONS}

In this paper, it has been shown that at P-band the dominant backscattering contribution varies significantly with the incidence angle. For a tropical forest, surface scattering prevails in near range and dihedral reflection in far range. At L-band, volume diffusion is much stronger and the dependence on the incidence angle is less pronounced.

Investigating the correlation between in-situ biomass measurements and the power of the decomposition components, it has been found that the dynamic range of the double-bounce component is increased with respect to the polarization channels and surface and volume scattering. The function for the relation between the dihedral component and biomass and the function of the relation between the volume diffusion contribution and biomass are rather different.
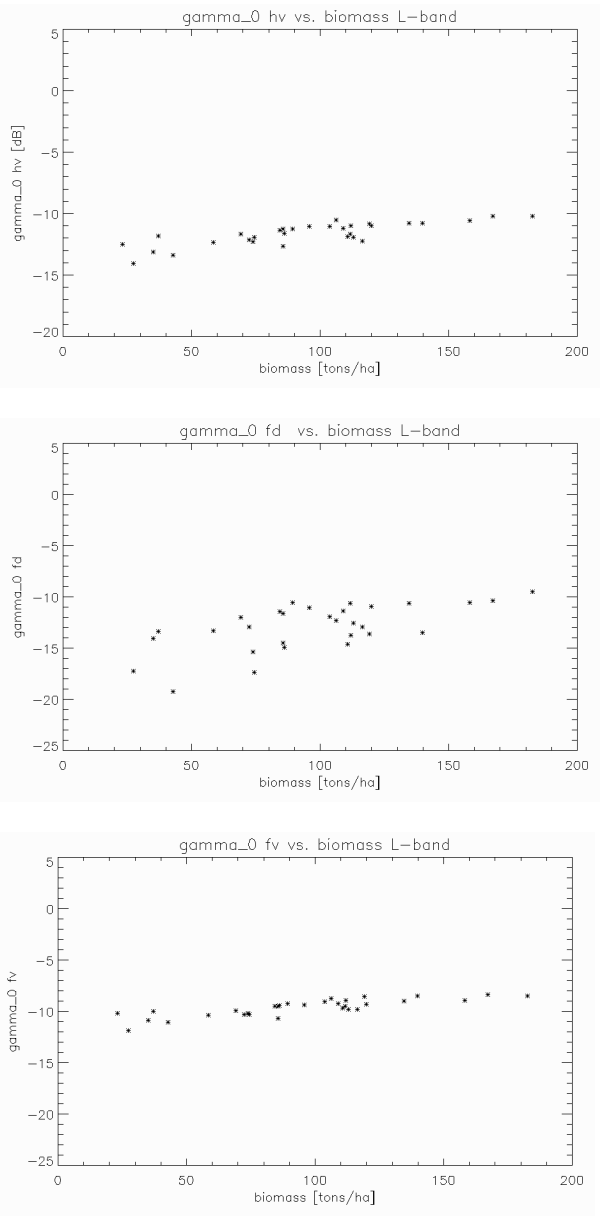

Fig. 11. Krycklan test site at L-band acquired from opposite viewing angle - $\gamma_{0}$ vs. biomass. Top: HV channel, middle: Dihedral, bottom: Volume diffusion.

\section{REFERENCES}

[1] T. Le Toan, A. Beaudoin, J. Riom, D. Guyon: Relating forest biomass to SAR data, IEEE Trans. Geoscience Remote Sensing, vol. 30, pp. 403-411, Feb. 1992.

[2] A. Beaudoin, T. Le Toan, et al.: Retrieval of forest biomass from SAR data, International Journal of Remote Sensing, vol. 15, pp. 2777-2796, 1994.

[3] S. R. Cloude: Polarisation applications in remote sensing, Oxford University Press, 2010.

[4] A. Freeman and S. L. Durden: A Three-Component Scattering Model for Polarimetric SAR Data, IEEE Trans. Geoscience and Remote Sensing, vol. 36, no. 3, pp. 963-973, May 1998. 